\title{
The Inhibition of CDK8/19 Mediator Kinases Prevents the Development of Resistance to EGFR-Targeting Drugs
}

\author{
Amanda C. Sharko ${ }^{1}$, Chang-Uk Lim ${ }^{1}$, Martina S. J. McDermott ${ }^{1}$, Chuck Hennes ${ }^{1}$, Kingsavanh P. Philavong ${ }^{1}$, \\ Tiffanie Aiken ${ }^{1}$, Victor V. Tatarskiy ${ }^{2}$, Igor B. Roninson ${ }^{1}$ (D) and Eugenia V. Broude ${ }^{1, *(D)}$ \\ 1 Department of Drug Discovery and Biomedical Sciences, University of South Carolina, \\ Columbia, SC 29208, USA; sharko@cop.sc.edu (A.C.S.); limc@cop.sc.edu (C.-U.L.); \\ martinamcdermott3@gmail.com (M.S.J.M.); ChuckHennes55@gmail.com (C.H.); \\ philavong.kp@gmail.com (K.P.P.); taiken0518@gmail.com (T.A.); roninsoni@cop.sc.edu (I.B.R.) \\ 2 Institute of Gene Biology, Russian Academy of Sciences, 119334 Moscow, Russia; tatarskii@gmail.com \\ * Correspondence: broude@cop.sc.edu; Tel.: +1-803-777-8440
}

\section{check for} updates

Citation: Sharko, A.C.; Lim, C.-U.; McDermott, M.S.J.; Hennes, C.;

Philavong, K.P.; Aiken, T.; Tatarskiy, V.V.; Roninson, I.B.; Broude, E.V. The Inhibition of CDK8/19 Mediator Kinases Prevents the Development of Resistance to EGFR-Targeting Drugs. Cells 2021, 10, 144. https://doi.org/ 10.3390/cells10010144

Received: 8 December 2020

Accepted: 9 January 2021

Published: 12 January 2021

Publisher's Note: MDPI stays neutral with regard to jurisdictional clai$\mathrm{ms}$ in published maps and institutional affiliations.

Copyright: (C) 2021 by the authors. Licensee MDPI, Basel, Switzerland. This article is an open access article distributed under the terms and conditions of the Creative Commons Attribution (CC BY) license (https:// creativecommons.org/licenses/by/ $4.0 /)$.

\begin{abstract}
Drug resistance is the main obstacle to achieving cures with both conventional and targeted anticancer drugs. The emergence of acquired drug resistance is initially mediated by non-genetic transcriptional changes, which occur at a much higher frequency than mutations and may involve population-scale transcriptomic adaptation. CDK8/19 kinases, through association with transcriptional Mediator complex, regulate transcriptional reprogramming by co-operating with different signal-responsive transcription factors. Here we tested if CDK8/19 inhibition could prevent adaptation to drugs acting on epidermal growth factor receptor (EGFR/ERBB1/HER1). The development of resistance was analyzed following long-term exposure of BT474 and SKBR3 breast cancer cells to EGFR-targeting small molecules (gefitinib, erlotinib) and of SW48 colon cancer cells to an anti-EGFR monoclonal antibody cetuximab. In all cases, treatment of small cell populations $\left(\sim 10^{5}\right.$ cells $)$ with a single dose of the drug initially led to growth inhibition that was followed by the resumption of proliferation and development of drug resistance in the adapted populations. However, this adaptation was always prevented by the addition of selective CDK8/19 inhibitors, even though such inhibitors alone had only moderate or no effect on cell growth. These results indicate that combining EGFR-targeting drugs with CDK8/19 inhibitors may delay or prevent the development of tumor resistance to therapy.
\end{abstract}

Keywords: CDK8; CDK19; EGFR; gefitinib; erlotinib; cetuximab; drug resistance

\section{Introduction}

Cancer drug resistance, a key obstacle to achieving cures, was long thought to result from the selection of rare preexisting tumor cells that carry genetic changes conferring resistance. In recent decades, however, it became clear that resistance to both conventional and targeted drugs involves metastable transcriptional changes that allow tumor cells to adapt and survive drug exposure [1-8]. While conclusions from different studies using the same drugs have varied in regard to the relative contributions of pre-existing genetic and stable epigenetic variants towards drug resistance [9-13], a consensus is emerging that transcriptomic plasticity of tumor cells and drug-induced adaptation is key to the initial emergence of low-level resistance. This non-genetic resistance of tumor cell populations provides the background for subsequent selection of stable genetic variants that yield higher levels of resistance $[5,13]$.

Most importantly from the therapeutic perspective, the induction of non-genetic resistance was found to be preventable pharmacologically, which has been first demonstrated by the ability of PKC/NFKB pathway inhibitors to prevent non-genetic induction of the multidrug transporter ABCB1 (MDR1/Pgp) [1,2]. In more recent examples, resistance to different classes of receptor tyrosine kinase inhibitors was reported to be prevented by 
THZ1, an inhibitor of transcriptional kinases CDK7, CDK12 and CDK13 [14], whereas a BRD9 inhibitor was shown to prevent resistance to EGFR-targeting agents [15] and a compound that prevented tamoxifen resistance was found to act by inhibiting NFKB [16]. However, agents that affect the general transcriptional machinery or inhibit basal NFKB activity are usually cytotoxic, which complicates both the study interpretation (independent cytotoxicity versus prevention of resistance) and the future clinical development.

We are exploring the potential utility of a new class of non-cytotoxic inhibitors of CDK8/19 Mediator kinases that regulate transcriptional reprogramming as novel resistancepreventing agents. CDK8 (ubiquitously expressed) and CDK19 (expressed in a tissuespecific manner) [17] are alternative components of the regulatory CDK8 module associated with the transcriptional Mediator complex; this module also includes cyclin C (CCNC, the binding partner of CDK8 and CDK19) and proteins MED12 and MED13 [18]. Unlike better-known CDKs (such as CDK2 or CDK4/6), CDK8/19 regulate transcription but not cell cycle progression [18,19]. Unlike other transcriptional CDKs, such as CDK7 or CDK9, they do not affect the overall transcription machinery. While CDK8 knockout is detrimental to embryonic development, a process that relies on transcriptional reprogramming [20-22], the knockout of CDK8 in adult mice has no apparent phenotypic consequences [23] and the knockdown or knockout of CDK8 or CDK19 [24] or pharmaceutical CDK8/19 kinase inhibitors [25-27] have little or no effect on the growth of most cell lines, in contrast to the effects of CDK7, CDK9 or cell cycle regulating CDKs [24]. Instead, CDK8/19 act as cofactors of several transcription factors, including $\beta$-catenin/TCF/LEF [28], SMADs [29,30], HIF1A [31], STATs [32], ER [33] and NFkB [34]; CDK8/19 inhibition decreases the induction of transcription of subsets of genes induced by the above transcription factors. CDK8/19 act both by direct phosphorylation of some transcription factors (e.g., SMADs, STATs) and also downstream of transcription factors by affecting (possibly indirectly) the phosphorylation of the C-terminal domain (CTD) of RNA polymerase II (Pol II), which allows the paused Pol II to detach from the promoter and complete transcription. The CTD phosphorylationbased mechanism was shown to be responsible for downstream potentiation of the serum response network [35], HIF1A [31], estrogen receptor (ER) [33] and NFKB [34] by CDK8/19. Importantly, CDK8/19 inhibition suppresses CTD phosphorylation not globally but only in the context of newly activated genes and CDK8/19 inhibitors suppress de novo induction of gene expression but not basal transcriptional activity [34]. This unique transcription regulatory pattern defines $C D K 8 / 19$ as regulators of transcriptional reprogramming [34].

The first evidence for the role of CDK8/19 in drug resistance came from a chemical genomics study where CDK8/19 were identified as the targets of senexin A and related small molecules that suppressed chemotherapy- or radiation-induced transcription of multiple paracrine factors that inhibit apoptosis and confer drug resistance [25]. These effects of CDK8/19 inhibitors were shown to be due to a large extent to the attenuation of NFKB-induced transcription [34]. In another study, we found that CDK8/19 inhibitors senexin A and senexin B prevent the development of estrogen independence in three different estrogen receptor-positive breast cancer cell lines during long-term estrogen deprivation [33].

In the present study, we tested the ability of highly selective chemically distinct CDK8/19 inhibitors senexin B $[34,36,37]$ and $15 \mathrm{w}[36,37]$ to prevent resistance to drugs targeting the epidermal growth factor receptor (EGFR/ERBB1/HER1). We have analyzed the development of resistance to small-molecule EGFR inhibitors gefitinib and erlotinib, which are used in non-small-cell lung cancers with mutated EGFR and that have been tested in other EGFR-driven tumors including breast cancers [38-41] and cetuximab, an anti-EGFR monoclonal antibody used for the treatment of colorectal cancer (with wildtype KRAS), non-small cell lung cancer and head and neck cancer [42]. Cancers treated with all the EGFR-targeted drugs eventually become resistant to them [43] indicating that prevention of such resistance is an important medical need. We have analyzed the effects of CDK8/19 inhibitors on the development of adaptive resistance in cell lines derived from breast cancer (gefitinib, erlotinib) and colon cancer (cetuximab). In all cases, the 
CDK8/19 inhibitors, while showing moderate or no growth-inhibitory activity when used alone, strongly suppressed the development of resistance to the EGFR-targeting agents. These results suggest that CDK8/19 inhibition may delay or prevent the development of resistance to EGFR-targeting drugs.

\section{Materials and Methods}

\subsection{Cell Lines and Reagents}

BT474, SKBR3 and SW48 cells were obtained from the American Type Culture Collection (Manassas, VA, USA). BT474 (ATCC: HTB-20) and SKBR3 (ATCC: HTB-30) cells were maintained, respectively, in RPMI-1640 and DMEM (ThermoFisher Scientific, Waltham, MA, USA) with 10\% FBS, 1\% penicillin-streptomycin and $2 \mathrm{mM}$ L-glutamine. SW48 (ATCC: CCL-231) were cultured in RPMI-1640 and 10\% FBS. Cells were routinely confirmed to be free of Mycoplasma (MycoAlert PLUS mycoplasma detection kit, Lonza, Walkersville, MD, USA) and were authenticated by STR profiling by the University of Arizona Genetic Core or by Source Bioscience in 2016. Gefitinib and erlotinib were purchased from LC Laboratories (Woburn, MA, USA), cetuximab (Erbitux) was from ImClone/Eli Lilly (Indianapolis, IN, USA), and senexin B and 15w from Senex Biotechnology (Columbia, SC, USA).

\subsection{Gefitinib and Erlotinib Selection and Sensitivity Testing}

To determine concentrations to select for acquired drug resistance, BT474 and SKBR3 breast cancer cells were seeded in 96-well plates (2500 and 1500 cells/well, respectively) and treated with gefitinib $(0-5 \mu \mathrm{M})$ and (in the case of BT474) with erlotinib $(0-10 \mu \mathrm{M})$ for 10 days. Visual assessment and image densitometry were used to determine the approximate minimum concentration necessary to produce maximum growth inhibition. Drug resistant cells were selected by plating 150,000 cells in T25 flasks and treating continuously with either erlotinib (BT474: $7.5 \mu \mathrm{M}$ ) or gefitinib (BT474: $1.25 \mu \mathrm{M}$; SKBR3: $2.0 \mu \mathrm{M})$, passaging as necessary. Cells were concurrently treated with erlotinib or gefitinib in combination with senexin B $(1 \mu \mathrm{M})$ or $15 \mathrm{w}(250 \mathrm{nM})$. Cell cultures were photographed in situ (Zeiss Axiovert 200; Carl Zeiss Microscopy, White Plains, NY, USA) before treatment, at 3 days, and then once weekly for 8-10 weeks after treatment initiation. Densitometric measurements were made using ImageJ software. Statistics were calculated by 2-way ANOVA with post-hoc multiple comparisons in Prism (GraphPad Software, San Diego, CA, USA). Representative flasks were also fixed, stained with crystal violet, and photographed at 2, 4, and 8-10 weeks after initiation of treatment.

For assessing drug resistance, BT474 and SKBR3 cells were seeded in 96-well plates (2500 and 1500 cells/well, respectively) and incubated overnight. Cells were then treated with DMSO (control), senexin B $(0-3 \mu \mathrm{M})$, erlotinib $(0-15 \mu \mathrm{M})$, or gefitinib $(0-3 \mu \mathrm{M})$ individually or in fixed ratio combinations (1:1 for gefitinib and senexin $B$ and 5:1 for erlotinib and senexin B). Cell growth was measured at 7 days after drug addition by MTT assay and expressed as a percentage of untreated cell signal. Synergy was assessed by calculating Combination Indices (CI values) using CompuSyn Software [44] and IC50 values were determined using the AAT Bioquest IC50 calculator (https://www.aatbio. com/tools/ic50-calculator/).

\subsection{Cetuximab Selection and Sensitivity Testing}

SW48 cells were seeded in 6-well plates (40,000 cells/well) and treated with cetuximab $(0-1 \mu \mathrm{M})$ and senexin B $(0.313-5 \mu \mathrm{M})$ for 7 days. The number of live cells was measured by flow cytometry using a LSR II system (Becton Dickinson, Franklin Lakes, NJ, USA) with a 96-well loader, after staining with $10 \mu \mathrm{g} / \mathrm{mL}$ propidium iodide. For long-term selection, parental SW48 cells were plated in T75 flasks (500,000 cells/flask) and then treated with vehicle (DMSO), or with cetuximab $(100 \mathrm{nM})$ and senexin B $(1 \mu \mathrm{M})$ alone and in combination. Cells (in duplicates) were collected, live cell numbers were measured by flow cytometry and cells were replated at the original density every 6-10 days for a total of 81 days. Changes in cell number over time were calculated relative to vehicle-treated cells 
using a multiplying factor (the ratio of the number of cells collected to the number plated at each passage).

\section{Results}

\subsection{Effects of CDK8/19 Inhibition on Gefitinib and Erlotinib Resistance}

We used breast cancer cell lines to analyze the effects of CDK8/19 inhibitor senexin B on the development of resistance to small-molecule EGFR inhibitors gefitinib and erlotinib. Single-step selections of limited duration (2-3 months) were carried out starting from $1.5 \times 10^{5}$ cells. This starting cell number and duration of exposure are too low to select drug-resistant mutants (see Discussion). Cells were plated in the presence of gefitinib or erlotinib at concentrations that produced near-maximum inhibition of cell growth after 10 days (see Materials and Methods). The results of representative single-step selections (from a total of three independent gefitinib and three erlotinib selections) conducted in BT474 breast cancer cells (ER- and HER2-positive) are shown in Figure 1. Figure 1A shows crystal violet staining of representative flasks at three time points of selection. Figure 1B shows phase contrast microscopic images of cells at multiple time points and Figure $1 \mathrm{C}$ presents densitometric measurements of microphotographs (four images per flask). After two weeks of continuous treatment, cells were nearly undetectable by crystal violet staining (Figure 1A) and very few cells were detectable microscopically (Figure 1B,C). After an additional two weeks of treatment (four weeks total), cells resumed proliferation and were actively growing at the 8-week time point, indicating that the cells had adapted to EGFR inhibitors.

To test the effect of CDK8/19 inhibition on the outcome of selection, we have used the compound senexin B (4-((2-(6-(4-methylpiperazine-1-carbonyl)naphthalen-2-yl)ethyl)amino) quinazoline-6-carbonitrile), which is highly selective for CDK8/19 based on the lack of off-target inhibition in extensive kinome profiling [45,46] and lack of phenotypic effects in CDK8/19 knockout cells [38,47]. In contrast, when selection was carried out in the presence of $1 \mu \mathrm{M}$ senexin B (concentration sufficient for near-maximum CDK8/19 kinase inhibition in cell-based assays [33,46]), cells did not grow out even after 8 weeks and were undetectable by crystal violet staining (Figure 1A) or showed minimal numbers by phase contrast microscopy (Figure 1B,C). To confirm the effects of CDK8/19 inhibition on the development of EGFR inhibitor resistance, we employed a chemically unrelated CDK8/19 inhibitor, 15w (3-amino-4-(4-(4-(2-(dimethylamino)-2-oxoethyl)phenyl)-1,4-diazepan-1-yl)thieno [2,3b]pyridine-2-carboxamide), which is also highly selective for CDK8/19 based on kinome profiling [36] and phenotypic analysis [37,46]. As with senexin B, the addition of $15 \mathrm{w}$ (used at $250 \mathrm{nM}$, due to its higher potency [38]) prevented the emergence of both gefitinib and erlotinib resistance, even after 8 weeks of treatment (Figure 1B,C), confirming that the resistance-preventing effect of senexin $B$ was mediated by CDK8/19 inhibition.

To confirm the observed effects in another cell line, we have used SKBR3 breast cancer cells (ER-negative, HER2-positive) for gefitinib selection, using the same study design as with BT474 cells. Figure 2 shows the results of a representative gefitinib selection (out of 4 independent selections). Gefitinib resistance took longer to develop in SKBR3 cells than in BT474, but by 10 weeks cells appeared fully adapted to the drug (Figure 2A-C). As with BT474 cells, the development of resistance in SKBR3 cells was fully prevented by the addition of different CDK8/19 inhibitors, senexin B and 15w (Figure 2A-C). 


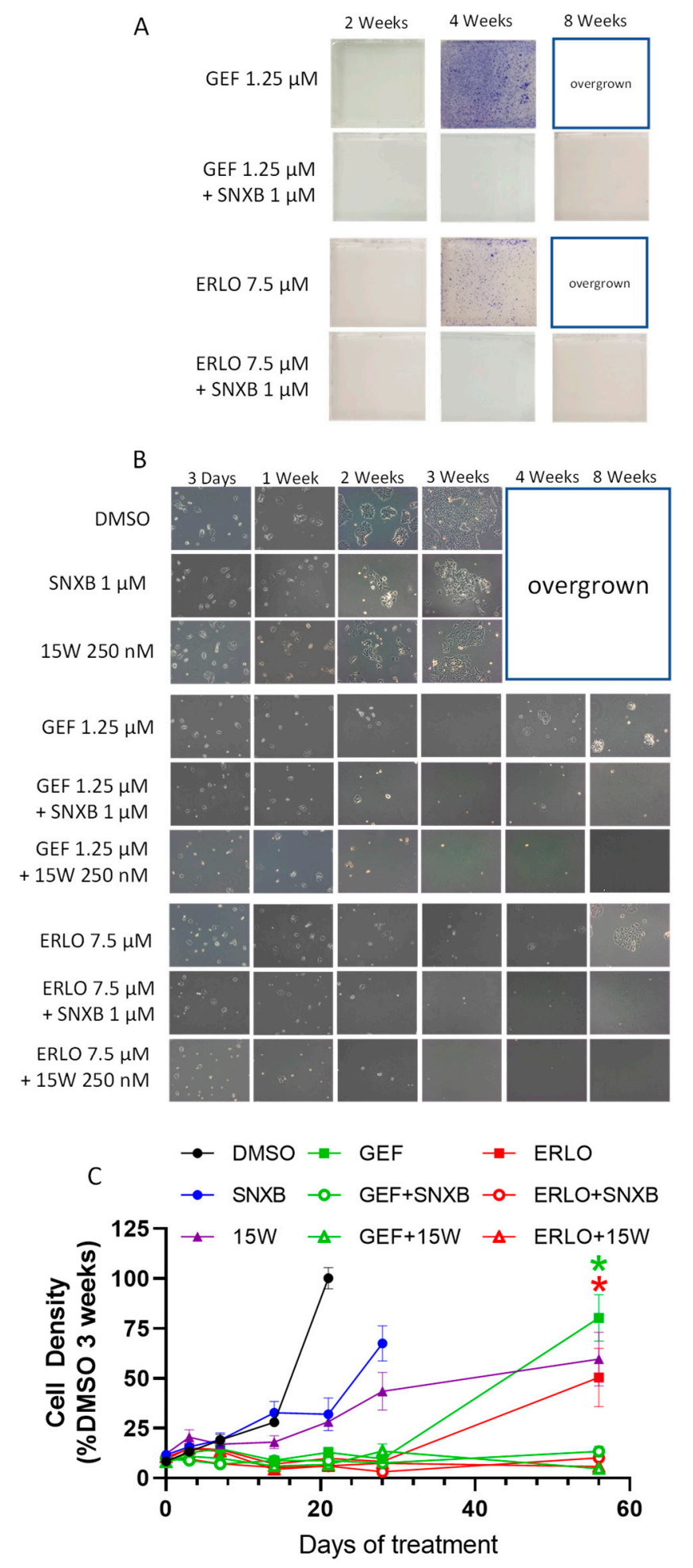

Figure 1. CDK8/19 inhibitors senexin B (SNXB) and 15w prevent resistance to EGFR inhibitors gefitinib (GEF) and erlotinib (ERLO) in BT474 breast cancer cells. (A). Representative photographs of crystal violet-stained flasks at 2, 4, and 8 weeks of treatment. (B). Representative phase-contrast microphotographs at 3 days, and at 1, 2, 3, 4 and 8 weeks of treatment. (C). Densitometric measurements of microphotographs expressed as percentage of cell density in DMSO controls at 3 weeks. Data shown as mean ( $n=4$ images /flask) \pm SEM. $p<0.0001$ for GEF vs. GEF+SNXB $/ 15 w\left(^{*}\right)$ and ERLO vs. ERLO+SNXB $/ 15 w\left({ }^{*}\right)$ at 8 weeks. 

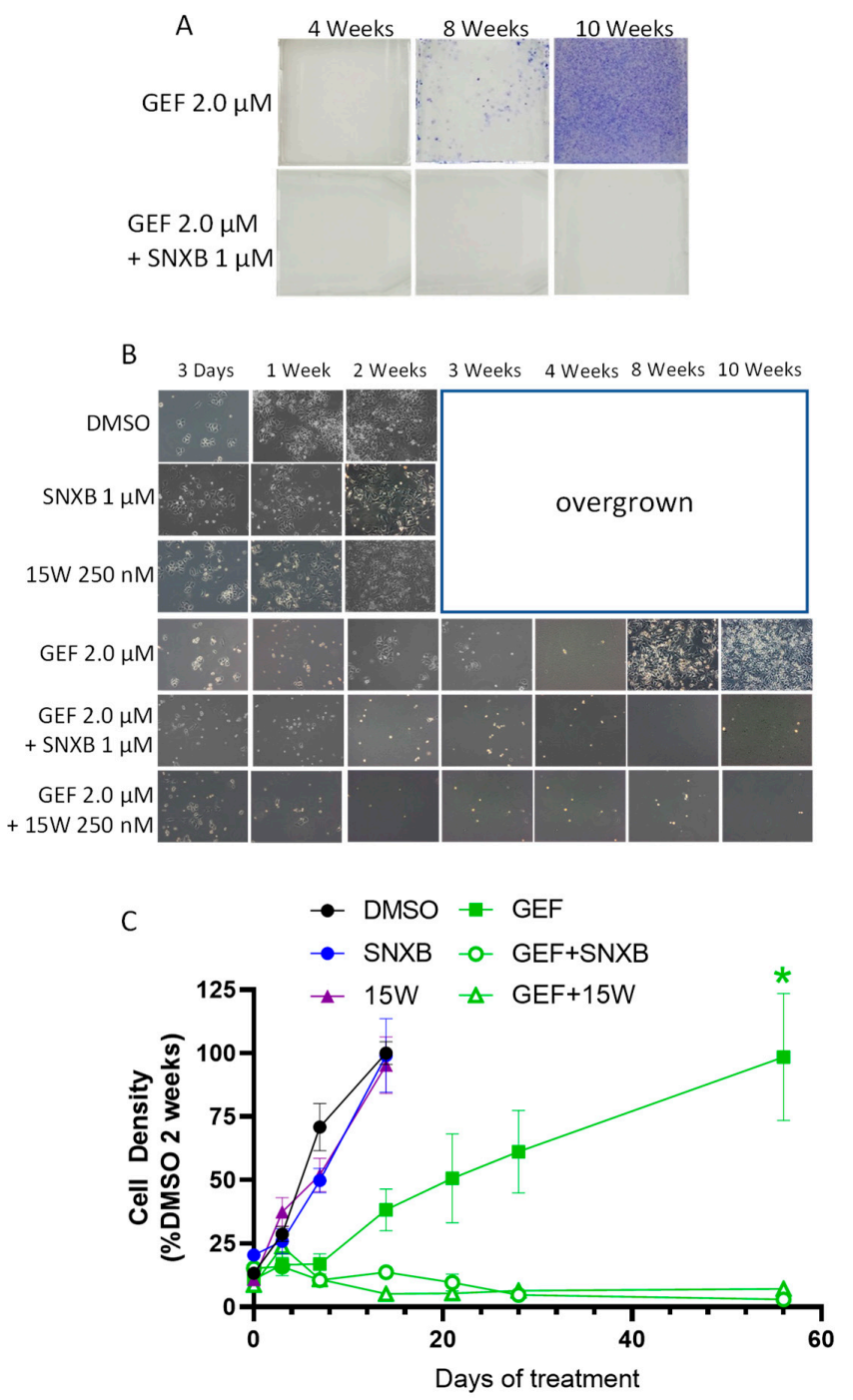

Figure 2. CDK8/19 inhibitors senexin B (SNXB) and 15w prevent resistance to EGFR inhibitor gefitinib (GEF) in SKBR3 breast cancer cells. (A). Representative photographs showing cell density (crystal violet staining) in flasks at 4, 8 and 10 weeks of treatment. (B). Representative phase-contrast microphotographs at 3 days, and at 1, 2, 3, 4, 8 and 10 weeks of treatment. (C). Densitometric measurements of photomicrographs expressed as percentage of cell density in DMSO controls at 2 weeks. Data shown as mean ( $n=4$ images /flask) \pm SEM. $p<0.0001$ for GEF vs. GEF+SNXB/15w $\left.{ }^{*}\right)$ at 8 weeks.

We have asked if the prevention of gefitinib and erlotinib resistance by CDK8/19 inhibitors could be due either to synergy between EGFR-targeting drugs and CDK8/19 inhibitors or to the reversal of acquired resistance to gefitinib or erlotinib. Synergy analysis was carried out by the Chou-Talalay method [44], which compares the effects of different concentrations of drugs (gefitinib or erlotinib and senexin B) used individually or at fixed-ratio combinations. In this method, the drug interactions are characterized by the Combination Index $(\mathrm{CI})$, where a synergistic interaction is defined by $\mathrm{CI}<1$. To determine if CDK8/19 inhibitor reversed the resistance acquired under our conditions, the same analysis was carried out on the gefitinib- or erlotinib-adapted cell populations, and the degrees of resistance to individual drugs and their combinations were determined by comparing IC50 values between the unselected and drug-adapted populations.

The analysis of gefitinib/senexin B interactions in BT474 cells is shown in Figure 3A-C and Table 1. Figure 3A shows the results of a 7-day growth inhibition assay of BT474 cells treated with gefitinib, senexin $B$, or their 1:1 combination. IC50 values measured in these 
assays are shown in Table 1 and CI values (determined at IC50 levels) are indicated in the graphs. Figure 3B,C and Table 1 show the results of the same analysis carried out with cells that were adapted to gefitinib (Figure 3B) or erlotinib (Figure 3C). Both gefitinib- and erlotinib-adapted BT474 cells showed increased gefitinib resistance (7.0-fold and 5.9-fold increase in IC50 relative to unselected cells, respectively). The addition of senexin B did not reverse resistance to EGFR inhibitors, as the resistant cells showed the same increase in IC50 values for gefitinib + senexin B combination relative to parental cells (5.9-fold for gefitinib-adapted and 7.0-fold for erlotinib-adapted cells). No significant synergy between gefitinib and senexin $B$ was observed in parental cells or in cells selected with gefitinib or erlotinib $(\mathrm{CI}=1.18,1.13$ and 0.91 , respectively). These results indicate that the resistancepreventing effect of the $\mathrm{CDK} 8 / 19$ inhibitor was due neither to overcoming resistance that has already emerged nor to a synergistic interaction between the compounds.
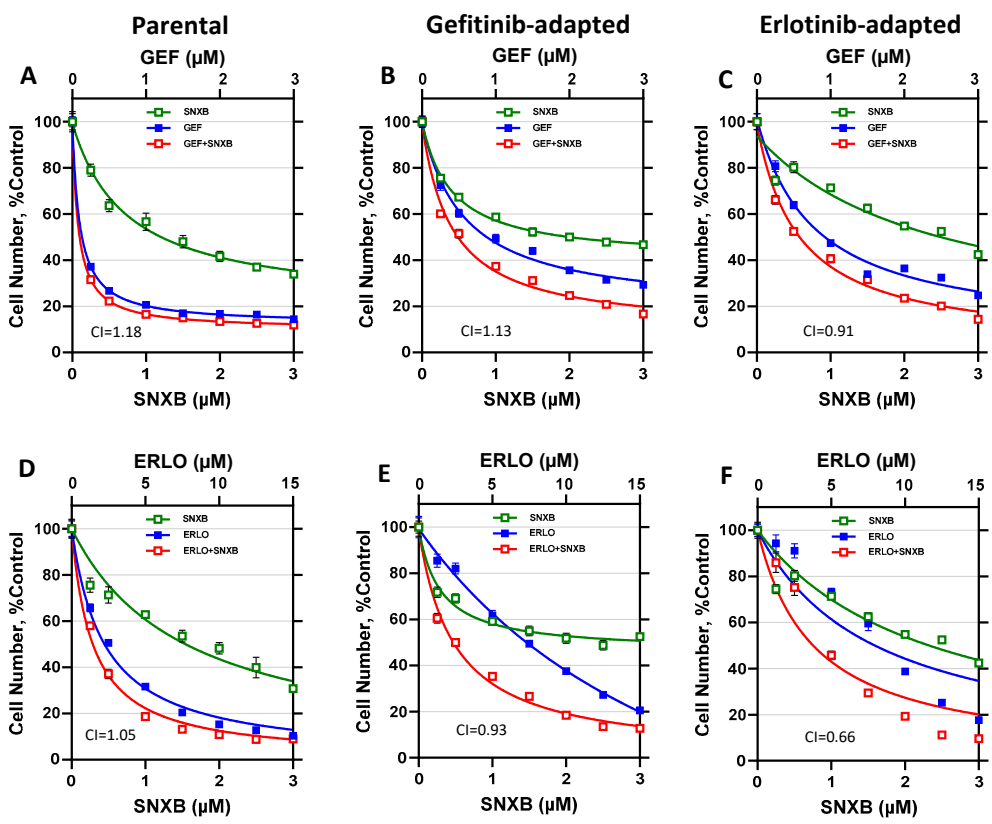

Figure 3. Effects of gefitinib and erlotinib alone and in combination with Senexin B in parental and gefitinib- or erlotinibadapted BT474 cells. (A-C). Effects of 7-day treatment with gefitinib (GEF) and senexin B (SNXB), alone or in 1:1 combination, in parental (A), gefitinib-adapted (B), and erlotinib-adapted (C) BT474 cells. (D-F). Effects of 7-day treatment with erlotinib (ERLO) and senexin B, alone or in 5:1 combination, in parental (D), gefitinib-adapted (E), and erlotinib-adapted (F) BT474 cells. Data shown as mean \pm SEM of six replicate measurements. Combination index (CI) values at IC50 concentrations are shown in lower left corner of each graph.

Table 1. $\mathrm{IC}_{50}$ values $(\mu \mathrm{M})$ for parental and gefitinib- or erlotinib-adapted cell populations in 7-day growth inhibition assays shown in Figures 3 and 4 . In case of drug combinations, IC $_{50}$ values are shown for each drug; fold change in $\mathrm{IC}_{50}$ relative to parental cells shown in parentheses (italicized). IC50 values for senexin B in SKBR3 cells could not be determined due to the low extent of growth inhibition.

\begin{tabular}{ccccccc}
\hline & BT474-Par & BT474-GefR & BT474-ErlR & SKBR3-Par & SKBR3-GefR \\
\hline Gefitinib & 0.140 & $0.973(6.95)$ & $0.863(6.16)$ & 0.619 & $1.530(2.47)$ \\
\hline Senexin B & 1.547 & $2.430(1.57)$ & $2.561(1.66)$ & N/A & N/A \\
\hline $\begin{array}{c}\text { Senexin B + } \\
\text { Gefitinib (1:1) }\end{array}$ & $0.087 / 0.087$ & $0.510 / 0.510(5.86)$ & $0.612 / 0.612(7.03)$ & 0.403 & $0.644(1.60)$ \\
\hline Erlotinib & 2.489 & $7.329(2.94)$ & $8.520(3.42)$ & 2.478 & $3.437(1.39)$ \\
\hline $\begin{array}{c}\text { Senexin B + } \\
\text { Erlotinib (1:5) }\end{array}$ & $0.351 / 1.753$ & $0.473 / 2.124(1.35)$ & $0.915 / 4.577(2.61)$ & $0.258 / 1.292$ & $0.328 / 1.641(1.27)$ \\
\hline
\end{tabular}



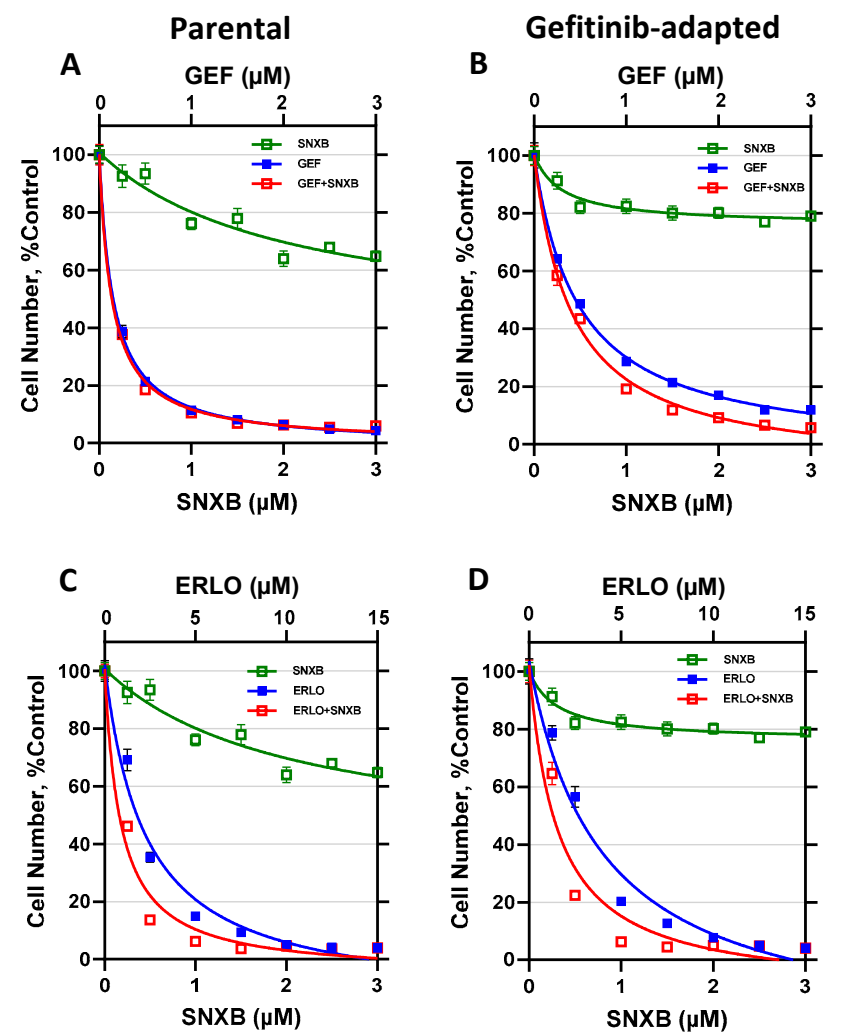

Figure 4. Effects of gefitinib and erlotinib alone and in combination with senexin $B$ in parental and gefitinib-adapted SKBR3 cells. (A,B). Effects of 7-day treatment with gefitinib and senexin B, alone or in 1:1 combination, in parental (A) and gefitinib-adapted (B) SKBR3 cells. (C,D). Effects of 7-day treatment with erlotinib and senexin $B$, alone or in 5:1 combination, in parental (C) and gefitinib-adapted (D) SKBR3 cells. Data shown as mean \pm SEM of six replicate measurements.

The same analysis was carried out for erlotinib/senexin B interactions in BT474 cells. Figure 3D-F and Table 1 show the response of parental and drug-adapted BT474 cells to erlotinib, senexin B or their 5:1 combination. Both erlotinib- and gefitinib-adapted BT474 cells were resistant to erlotinib alone (3.4- and 2.9-fold relative to parental cells, respectively) and to erlotinib+senexin B combination (2.6- and 1.4-fold, respectively). CI values showed no synergy between erlotinib and senexin $B$ for the parental and gefitinib-adapted cells (CI $=1.05$ and 0.93 , respectively) but this drug combination appeared synergistic $(\mathrm{CI}=0.66)$ in erlotinib-adapted cells. Together with the finding that erlotinib-adapted cells were less resistant to erlotinib + senexin B combination than to erlotinib alone, this result suggests that reversal of erlotinib resistance could have contributed to the prevention of erlotinib resistance by CDK8/19 inhibitors.

The same analysis for SKBR3 cells is shown in Figure 4 and Table 1. The effects of gefitinib and senexin B (Figure 4A,B) and erlotinib and senexin B (Figure 4C,D) are shown for the parental SKBR3 cells (Figure $4 A, C$ ) and their gefitinib-adapted population (Figure 4B,D). While senexin B showed significant growth inhibition in ER-positive BT474 cells (Figure 3), likely due to its activity as an inhibitor of estrogen signaling [33], the growth inhibitory effects of senexin B were very weak in ER-negative SKBR3 cells and never approached 50 percent. Hence, $\mathrm{CI}$ values at $\mathrm{IC}_{50}$ for the combinations of EGFR and CDK8/19 inhibitors could not be determined in SKBR3 cells. Gefitinib-adapted SKBR3 cells showed a moderate increase in their $\mathrm{IC}_{50}$ values for gefitinib (2.5-fold) and erlotinib (1.4fold), whereas the increase in resistance to the combinations of these drugs with senexin $B$ was 1.6-fold and 1.3-fold, respectively. 


\subsection{Effects of CDK8/19 Inhibition on Cetuximab Resistance}

Cetuximab selection was carried out in SW48 colon carcinoma cells (wild-type KRAS), using $100 \mathrm{nM}$ cetuximab. As shown in Figure 5A, this concentration decreases the number of live (propidium iodide (PI)-negative) SW48 cells to $30.9 \%$ of untreated cells after 7-day treatment, whereas the dead (PI-positive) cell fraction was below $3 \%$, indicating that the effect of cetuximab is primarily cytostatic. The addition of senexin $B$ at concentrations below $2.5 \mu \mathrm{M}$ had little effect on 7-day survival of cetuximab (Figure 5A). For long-term selection, $5 \times 10^{5}$ SW48 cells were treated (i) with vehicle (DMSO) control, (ii) $1 \mu \mathrm{M}$ senexin $\mathrm{B}$, (iii) $100 \mathrm{nM}$ cetuximab, or (iv) $100 \mathrm{nM}$ cetuximab $+1 \mu \mathrm{M}$ senexin B. Cells were replated and the number of live cells was determined (in duplicates) by flow cytometry every 6-10 days, for a total of 81 days. Changes in cell number (calculated at each time point taking dilutions into account; see Materials and Methods) relative to the control cells are plotted in Figure 5B.

A

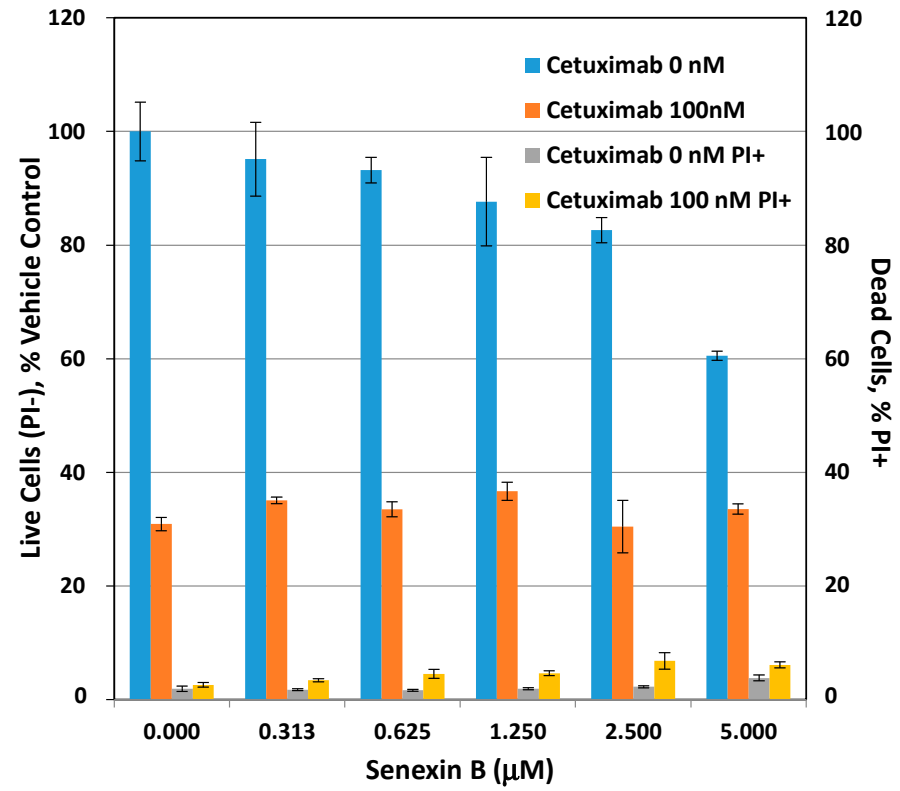

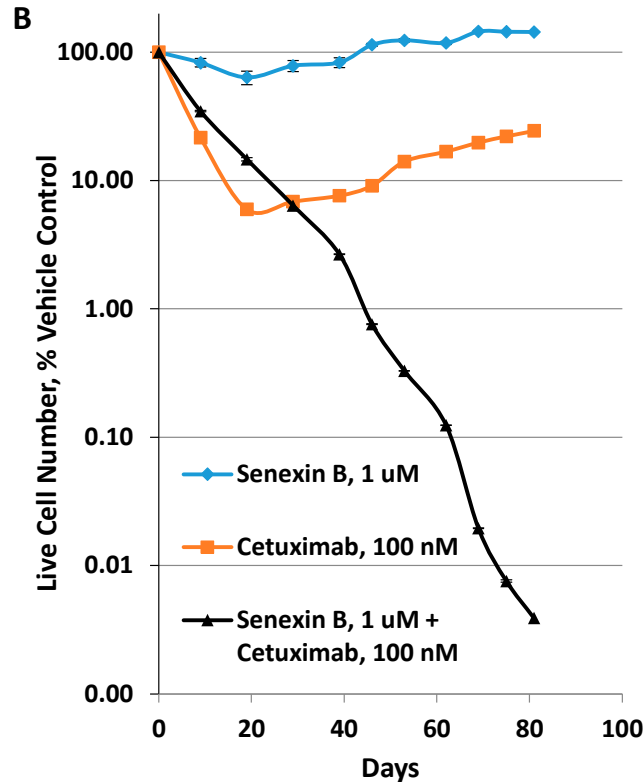

Figure 5. Effects of senexin B on the development of cetuximab resistance in SW48 colon carcinoma cells. (A) Effects of 7-day treatment with the indicated concentrations of Senexin B combined with vehicle or $100 \mathrm{nM}$ cetuximab on the number of live (PI-) cells relative to vehicle control and the fraction of dead (PI+) cells. (B) Effects of prolonged treatment with senexin B $(1 \mu \mathrm{M})$, cetuximab $(100 \mathrm{nM})$ or senexin B plus cetuximab combination. Cell numbers for each time point are shown relative to untreated cells (mean \pm SEM of duplicate measurements; error bars at most points are smaller than symbol size).

Senexin B alone had no significant effect on cell growth over time. $100 \mathrm{nM}$ cetuximab was growth-inhibitory for the first 19 days of treatment but after that point cetuximab no longer inhibited cell growth and the cells continued to grow until the end of the study (81 days), at a rate that was even higher than the control (marked by the slope of the curve), indicating the development of a cetuximab-resistant phenotype. The addition of senexin B initially decreased response to cetuximab, suggesting an apparently antagonistic interaction until the time when cells treated with cetuximab alone developed resistance. In contrast, the combination of cetuximab with the CDK8/19 inhibitor continued to inhibit cell growth until the end of the study (day 81), with no apparent development of resistance. The difference in calculated cell numbers between cetuximab alone and cetuximab + senexin B treatments exceeded four orders of magnitude starting between days 62 and 69 . Therefore, despite the apparent antagonism in the short-term, the addition of CDK8/19 inhibitor greatly improves the outcome of cetuximab treatment in the long-term, by preventing the development of cetuximab resistance. 


\section{Discussion}

In the present study, we have analyzed the effects of CDK8/19 inhibition on the development of adaptive resistance to EGFR-targeting small molecules (gefitinib and erlotinib) or an antibody (cetuximab). We have carried out single-step selection for resistance to EGFR-targeting drugs by plating a relatively small cell number $\left(1.5 \times 10^{5}\right.$ cells for BT474 and SKBR3 and $5 \times 10^{5}$ cells for SW48 cells) and using relatively short periods of drug exposure (up to 10 weeks). According to recent analysis [47] mutation rates per base per cell division in cancer are on the order of $10^{-8}-10^{-7}$ and a minimum of $2 \times 10^{7}$ cells (10 T175 flasks) would be required to have even a single mutant; it also takes 6-12 months of selection to achieve full drug resistance. Nevertheless, in our study, resistance in all the cell/drug combinations developed in every flask in less than 10 weeks, suggesting that such resistance represented drug adaptation of cell populations and was not mediated by genetic mechanisms. In all the tested cell-drug combinations, the development of this adaptive resistance was prevented by a highly selective CDK8/19 inhibitor senexin $\mathrm{B}$. The role of CDK8/19 in the development of resistance was verified through the use of another highly selective CDK8/19 inhibitor, $15 \mathrm{w}$, which bears no structural similarity to senexin B. To simplify future analysis of the role of CDK8/19 Mediator kinase in drug resistance, we are currently generating a series of tumor cell lines with CRISPR/CAS9 knockout of CDK8 and CDK19, followed by re-expression of the wild-type Mediator kinases and their kinase-dead mutants.

Although CDK8/19 inhibitors consistently prevented the development of adaptive resistance to EGFR-targeting drugs in all four tested cell line/EGFR inhibitor combinations, this effect in most cases could not be attributed to drug synergy or to reversal of resistance. In particular, gefitinib-adapted BT474 cells showed the same increase in resistance to gefitinib or erlotinib as to combinations of these drugs with senexin $B$, although erlotinibadapted cells were less resistant to the combination of erlotinib with senexin $B$ than to erlotinib alone. No synergy was apparent in gefitinib or erlotinib combinations with senexin $B$ (in contrast to a strong synergy found between erlotinib and THZ1, an inhibitor of CDK7/CDK12/CDK13 [48]). In the course of cetuximab selection, the addition of senexin B even decreased the response to cetuximab in the short-term but nevertheless prevented the development of cetuximab resistance. Hence, the observed prevention of resistance most likely reflects the inhibition of CDK8/19-mediated transcriptional reprogramming $[18,34]$ that prevents the emergence of adaptive resistance to EGFR-targeting drugs.

Numerous mechanisms of resistance to EGFR inhibitors have been described [42,44,49,50]. These include activating mutations in EGFR itself, mutations of BRAF, KRAS and PIK3CA, as well as amplification or non-genetic upregulation of HER2 and MET, and downregulation or mutation of PTEN. The resistance was also associated with the activation of various compensatory pathways, such as Wnt/ $\beta$-catenin and NFkB, and compensatory growth factor receptor signaling, including overexpression of HER family ligands, as well as HGF and FGF. Epithelial-mesenchymal transition (EMT) has also been identified as a major mechanism of resistance to EGFR-targeting drugs [49]. As discussed above, the small cell number used in our selections and the observed rapid development of resistance argue against the involvement of any genetic mechanisms. In addition, cross-resistance of gefitinib- and erlotinib-adapted cells argues against the involvement of inhibitor-specific mutations in the EGFR gene. Since drug-resistant cell populations were isolated here via rapid single-step adaptation, they almost certainly represent mixtures of cells with different transcriptomic mechanisms of resistance. With the advent of single-cell (sc) RNA-Seq, it became clear that adaptive drug resistance involves rapid transcriptional changes, which are observed concurrently in the same cell population [5-7,50]. In particular, a heterogeneous pattern of resistance-associated transcriptional changes was observed in scRNA-Seq analysis of the effects of short-term (5-day) cetuximab treatment in three head and neck squamous cell carcinoma cell lines [51]. Transcriptomic heterogeneity within the same cell lines was found to increase as an immediate response to cetuximab, and this effect was suggested to indicate that different cell subclones in the same cell line are activating alter- 
native pathways to overcome EGFR inhibition [51]. In the future, we are planning to use scRNA-Seq to identify the spectrum of CDK8/19-dependent transcriptomic mechanisms that lead to non-genetic resistance to EGFR-targeting drugs.

We have previously reported that CDK8/19 inhibitors prevent the induction of resistance-associated anti-apoptotic factors upon treatment with DNA-damaging drugs or ionizing radiation [25] and suppress the emergence of estrogen-independent ER-positive breast cancer cells upon prolonged estrogen deprivation [33]. These results, combined with the present study, indicate a pleiotropic effect of CDK8/19 on the emergence of drug resistance and suggest potential utility of CDK8/19 inhibitors in preventing the development of resistance to different classes of drugs.

Author Contributions: Conceptualization, E.V.B., I.B.R., M.S.J.M., A.C.S., C.-U.L.; methodology, A.C.S., M.S.J.M., C.-U.L., E.V.B.; formal analysis, A.C.S., C.-U.L.; investigation, A.C.S., C.-U.L., M.S.J.M., C.H., T.A., K.P.P., V.V.T.; data curation, A.C.S., C.-U.L.; writing-original draft preparation, E.V.B., I.B.R., A.C.S.; writing-review and editing, A.C.S., E.V.B., I.B.R.; supervision, E.V.B., I.B.R.; funding acquisition, V.V.T., E.V.B., I.B.R. All authors have read and agreed to the published version of the manuscript.

Funding: This research was funded by NIH grants P20 GM109091 (EVB, IBR) and P30GM103336 (EVB) and Megagrant (Agreement No.14.W03.31.0020 between the Ministry of Science and Education of the Russian Federation and Institute of Gene Biology, Russian Academy of Sciences) (IBR, VVT), and Gateway Research Scholarship from the American Foundation for Pharmaceutical Education (KPP).

Acknowledgments: We thank Microscopy and Flow Cytometry Core of the COBRE Center for Targeted Therapeutics, supported by NIH COBRE grant P20 GM109091, for assistance with flow cytometry and microscopy.

Conflicts of Interest: I.B.R. is Founder and President and E.V.B. is a consultant of Senex Biotechnology Inc.; other authors declare no conflict of interest.

\section{References}

1. Chaudhary, P.M.; Roninson, I.B. Induction of multidrug resistance in human cells by transient exposure to different chemotherapeutic drugs. J. Natl. Cancer Inst. 1993, 85, 632-639. [CrossRef]

2. Shtil, A.A. Signal transduction pathways and transcriptional mechanisms as targets for prevention of emergence of multidrug resistance in human cancer cells. Curr. Drug Targets 2001, 2, 57-77. [CrossRef]

3. Wang, J.; Seebacher, N.; Shi, H.; Kan, Q.; Duan, Z. Novel strategies to prevent the development of multidrug resistance (MDR) in cancer. Oncotarget 2017, 8, 84559-84571. [CrossRef] [PubMed]

4. Pisco, A.O.; Brock, A.; Zhou, J.; Moor, A.; Mojtahedi, M.; Jackson, D.; Huang, S. Non-Darwinian dynamics in therapy-induced cancer drug resistance. Nat. Commun. 2013, 4, 2467. [CrossRef] [PubMed]

5. Salgia, R.; Kulkarni, P. The Genetic/Non-genetic Duality of Drug 'Resistance' in Cancer. Trends Cancer 2018, 4, 110-118. [CrossRef] [PubMed]

6. Kyrochristos, I.D.; Ziogas, D.E.; Roukos, D.H. Drug resistance: Origins, evolution and characterization of genomic clones and the tumor ecosystem to optimize precise individualized therapy. Drug Discov. Today 2019, 24, 1281-1294. [CrossRef] [PubMed]

7. Hammerlindl, H.; Schaider, H. Tumor cell-intrinsic phenotypic plasticity facilitates adaptive cellular reprogramming driving acquired drug resistance. J. Cell Commun. Signal. 2018, 12, 133-141. [CrossRef]

8. Wang, Q.Y.; Zhong, H. Epigenetic programming contributes to development of drug resistance in hematological malignancies. Front. Biosci. (Landmark Ed.) 2015, 20, 728-742.

9. Shaffer, S.M.; Dunagin, M.C.; Torborg, S.R.; Torre, E.A.; Emert, B.; Krepler, C.; Beqiri, M.; Sproesser, K.; Brafford, P.A.; Xiao, M.; et al. Rare cell variability and drug-induced reprogramming as a mode of cancer drug resistance. Nature 2017, 546, 431-435. [CrossRef]

10. Arozarena, I.; Wellbrock, C. Phenotype plasticity as enabler of melanoma progression and therapy resistance. Nat. Rev. Cancer 2019, 19, 377-391. [CrossRef]

11. Fallahi-Sichani, M.; Becker, V.; Izar, B.; Baker, G.J.; Lin, J.R.; Boswell, S.A.; Shah, P.; Rotem, A.; Garraway, L.A.; Sorger, P.K. Adaptive resistance of melanoma cells to RAF inhibition via reversible induction of a slowly dividing de-differentiated state. Mol. Syst. Biol. 2017, 13, 905. [CrossRef] [PubMed]

12. Su, Y.; Wei, W.; Robert, L.; Xue, M.; Tsoi, J.; Garcia-Diaz, A.; Homet Moreno, B.; Kim, J.; Ng, R.H.; Lee, J.W.; et al. Single-cell analysis resolves the cell state transition and signaling dynamics associated with melanoma drug-induced resistance. Proc. Natl. Acad. Sci. USA 2017, 114, 13679-13684. [CrossRef] [PubMed] 
13. Marine, J.C.; Dawson, S.J.; Dawson, M.A. Non-genetic mechanisms of therapeutic resistance in cancer. Nat. Rev. Cancer 2020. [CrossRef] [PubMed]

14. Rusan, M.; Li, K.; Li, Y.; Christensen, C.L.; Abraham, B.J.; Kwiatkowski, N.; Buczkowski, K.A.; Bockorny, B.; Chen, T.; Li, S.; et al. Suppression of Adaptive Responses to Targeted Cancer Therapy by Transcriptional Repression. Cancer Discov. 2018, 8, 59-73. [CrossRef] [PubMed]

15. Crawford, T.D.; Vartanian, S.; Cote, A.; Bellon, S.; Duplessis, M.; Flynn, E.M.; Hewitt, M.; Huang, H.R.; Kiefer, J.R.; Murray, J.; et al. Inhibition of bromodomain-containing protein 9 for the prevention of epigenetically-defined drug resistance. Bioorganic Med. Chem. Lett. 2017, 27, 3534-3541. [CrossRef] [PubMed]

16. Jin, X.H.; Jia, Y.S.; Shi, Y.H.; Li, Q.Y.; Bao, S.Q.; Lu, W.P.; Tong, Z.S. ACT001 can prevent and reverse tamoxifen resistance in human breast cancer cell lines by inhibiting NF-kappaB activation. J. Cell. Biochem. 2018. [CrossRef]

17. Tsutsui, T.; Fukasawa, R.; Tanaka, A.; Hirose, Y.; Ohkuma, Y. Identification of target genes for the CDK subunits of the Mediator complex. Genes Cells 2011, 16, 1208-1218. [CrossRef]

18. Fant, C.B.; Taatjes, D.J. Regulatory functions of the Mediator kinases CDK8 and CDK19. Transcription 2019, 10, 76-90. [CrossRef]

19. Philip, S.; Kumarasiri, M.; Teo, T.; Yu, M.; Wang, S. Cyclin-Dependent Kinase 8: A New Hope in Targeted Cancer Therapy? J. Med. Chem. 2018. [CrossRef]

20. Westerling, T.; Kuuluvainen, E.; Makela, T.P. Cdk8 is essential for preimplantation mouse development. Mol. Cell Biol. 2007, 27, 6177-6182. [CrossRef]

21. Lynch, C.J.; Bernad, R.; Martínez-Val, A.; Shahbazi, M.N.; Nóbrega-Pereira, S.; Calvo, I.; Blanco-Aparicio, C.; Tarantino, C.; Garreta, E.; Richart-Ginés, L.; et al. Global hyperactivation of enhancers stabilizes human and mouse naive pluripotency through inhibition of CDK8/19 Mediator kinases. Nat. Cell Biol. 2020, 22, 1223-1238. [CrossRef]

22. Postlmayr, A.; Dumeau, C.E.; Wutz, A. Cdk8 is required for establishment of H3K27me3 and gene repression by Xist and mouse development. Development 2020, 147. [CrossRef] [PubMed]

23. Adler, A.S.; McCleland, M.L.; Truong, T.; Lau, S.; Modrusan, Z.; Soukup, T.M.; Roose-Girma, M.; Blackwood, E.M.; Firestein, R. CDK8 maintains tumor dedifferentiation and embryonic stem cell pluripotency. Cancer Res. 2012, 72, 2129-2139. [CrossRef] [PubMed]

24. Roninson, I.B.; Győrffy, B.; Mack, Z.T.; Shtil, A.A.; Shtutman, M.S.; Chen, M.; Broude, E.V. Identifying Cancers Impacted by CDK8/19. Cells 2019, 8, 821. [CrossRef] [PubMed]

25. Porter, D.C.; Farmaki, E.; Altilia, S.; Schools, G.P.; West, D.K.; Chen, M.; Chang, B.D.; Puzyrev, A.T.; Lim, C.U.; Rokow-Kittell, R.; et al. Cyclin-dependent kinase 8 mediates chemotherapy-induced tumor-promoting paracrine activities. Proc. Natl. Acad. Sci. USA 2012, 109, 13799-13804. [CrossRef]

26. Koehler, M.F.; Bergeron, P.; Blackwood, E.M.; Bowman, K.; Clark, K.R.; Firestein, R.; Kiefer, J.R.; Maskos, K.; McCleland, M.L.; Orren, L.; et al. Development of a Potent, Specific CDK8 Kinase Inhibitor Which Phenocopies CDK8/19 Knockout Cells. Acs Med. Chem. Lett. 2016, 7, 223-228. [CrossRef]

27. Liang, J.; Chen, M.; Hughes, D.; Chumanevich, A.A.; Altilia, S.; Kaza, V.; Lim, C.U.; Kiaris, H.; Mythreye, K.; Pena, M.M.; et al. CDK8 Selectively Promotes the Growth of Colon Cancer Metastases in the Liver by Regulating Gene Expression of TIMP3 and Matrix Metalloproteinases. Cancer Res. 2018, 78, 6594-6606. [CrossRef]

28. Firestein, R.; Bass, A.J.; Kim, S.Y.; Dunn, I.F.; Silver, S.J.; Guney, I.; Freed, E.; Ligon, A.H.; Vena, N.; Ogino, S.; et al. CDK8 is a colorectal cancer oncogene that regulates beta-catenin activity. Nature 2008, 455, 547-551. [CrossRef]

29. Alarcon, C.; Zaromytidou, A.I.; Xi, Q.; Gao, S.; Yu, J.; Fujisawa, S.; Barlas, A.; Miller, A.N.; Manova-Todorova, K.; Macias, M.J.; et al. Nuclear CDKs drive Smad transcriptional activation and turnover in BMP and TGF-beta pathways. Cell 2009, 139, 757-769. [CrossRef]

30. Serrao, A.; Jenkins, L.M.; Chumanevich, A.A.; Horst, B.; Liang, J.; Gatza, M.L.; Lee, N.Y.; Roninson, I.B.; Broude, E.V.; Mythreye, K. Mediator kinase CDK8/CDK19 drives YAP1-dependent BMP4-induced EMT in cancer. Oncogene 2018, 37, 4792-4808. [CrossRef]

31. Galbraith, M.D.; Allen, M.A.; Bensard, C.L.; Wang, X.; Schwinn, M.K.; Qin, B.; Long, H.W.; Daniels, D.L.; Hahn, W.C.; Dowell, R.D.; et al. HIF1A employs CDK8-mediator to stimulate RNAPII elongation in response to hypoxia. Cell 2013, 153, 1327-1339. [CrossRef] [PubMed]

32. Bancerek, J.; Poss, Z.C.; Steinparzer, I.; Sedlyarov, V.; Pfaffenwimmer, T.; Mikulic, I.; Dolken, L.; Strobl, B.; Muller, M.; Taatjes, D.J.; et al. CDK8 kinase phosphorylates transcription factor STAT1 to selectively regulate the interferon response. Immunity 2013, 38, 250-262. [CrossRef] [PubMed]

33. McDermott, M.S.; Chumanevich, A.A.; Lim, C.U.; Liang, J.; Chen, M.; Altilia, S.; Oliver, D.; Rae, J.M.; Shtutman, M.; Kiaris, H.; et al. Inhibition of CDK8 mediator kinase suppresses estrogen dependent transcription and the growth of estrogen receptor positive breast cancer. Oncotarget 2017, 8, 12558-12575. [CrossRef] [PubMed]

34. Chen, M.; Liang, J.; Ji, H.; Yang, Z.; Altilia, S.; Hu, B.; Schronce, A.; McDermott, M.S.J.; Schools, G.P.; Lim, C.U.; et al. CDK8/19 Mediator kinases potentiate induction of transcription by NFkappaB. Proc. Natl. Acad. Sci. USA 2017, 114, 10208-10213. [CrossRef] [PubMed]

35. Donner, A.J.; Ebmeier, C.C.; Taatjes, D.J.; Espinosa, J.M. CDK8 is a positive regulator of transcriptional elongation within the serum response network. Nat. Struct. Mol. Biol. 2010, 17, 194-201. [CrossRef] 
36. Amirhosseini, M.; Bernhardsson, M.; Lang, P.; Andersson, G.; Flygare, J.; Fahlgren, A. Cyclin-dependent kinase 8/19 inhibition suppresses osteoclastogenesis by downregulating RANK and promotes osteoblast mineralization and cancellous bone healing. $J$. Cell. Physiol. 2019. [CrossRef]

37. Li, J.; Ji, H.; Porter, D.C.; Broude, E.V.; Roninson, I.B.; Chen, M. Characterizing CDK8/19 Inhibitors through a NFkB-Dependent Cell-Based Assay. Cells 2019, 8, 1208. [CrossRef]

38. Kalykaki, A.; Agelaki, S.; Kallergi, G.; Xyrafas, A.; Mavroudis, D.; Georgoulias, V. Elimination of EGFR-expressing circulating tumor cells in patients with metastatic breast cancer treated with gefitinib. Cancer Chemother. Pharmacol. 2014, 73, 685-693. [CrossRef]

39. Dickler, M.N.; Cobleigh, M.A.; Miller, K.D.; Klein, P.M.; Winer, E.P. Efficacy and safety of erlotinib in patients with locally advanced or metastatic breast cancer. Breast Cancer Res. Treat. 2009, 115, 115-121. [CrossRef]

40. Ferrer-Soler, L.; Vazquez-Martin, A.; Brunet, J.; Menendez, J.A.; De Llorens, R.; Colomer, R. An update of the mechanisms of resistance to EGFR-tyrosine kinase inhibitors in breast cancer: Gefitinib (Iressa) -induced changes in the expression and nucleo-cytoplasmic trafficking of HER-ligands (Review). Int. J. Mol. Med. 2007, 20, 3-10. [CrossRef]

41. Al-Mahmood, S.; Sapiezynski, J.; Garbuzenko, O.B.; Minko, T. Metastatic and triple-negative breast cancer: Challenges and treatment options. Drug Deliv. Transl. Res. 2018, 8, 1483-1507. [CrossRef] [PubMed]

42. Brand, T.M.; Iida, M.; Wheeler, D.L. Molecular mechanisms of resistance to the EGFR monoclonal antibody cetuximab. Cancer Biol. 2011, 11, 777-792. [CrossRef] [PubMed]

43. Roskoski, R., Jr. The ErbB/HER family of protein-tyrosine kinases and cancer. Pharmacol. Res. 2014, 79, 34-74. [CrossRef] [PubMed]

44. Chou, T.C. Drug combination studies and their synergy quantification using the Chou-Talalay method. Cancer Res. 2010, 70, 440-446. [CrossRef]

45. Roninson, I.B.; Porter, D.C.; Wentland, M.P. CDK8-CDK19 Selective Inhibitors and Their Use in Anti-Metastatic and Chemopreventative Methods for Cancer. U.S. Patent US 09321737, 26 April 2016.

46. Chen, M.; Li, J.; Liang, J.; Thompson, Z.S.; Kathrein, K.; Broude, E.V.; Roninson, I.B. Systemic Toxicity Reported for CDK8/19 Inhibitors CCT251921 and MSC2530818 Is Not Due to Target Inhibition. Cells 2019, 8, 1413. [CrossRef]

47. Acar, A.; Nichol, D.; Fernandez-Mateos, J.; Cresswell, G.D.; Barozzi, I.; Hong, S.P.; Trahearn, N.; Spiteri, I.; Stubbs, M.; Burke, R.; et al. Exploiting evolutionary steering to induce collateral drug sensitivity in cancer. Nat. Commun 2020, 11, 1923. [CrossRef] [PubMed]

48. McDermott, M.S.J.; Sharko, A.C.; Munie, J.; Kassler, S.; Melendez, T.; Lim, C.U.; Broude, E.V. CDK7 Inhibition is Effective in all the Subtypes of Breast Cancer: Determinants of Response and Synergy with EGFR Inhibition. Cells 2020, 9, 638. [CrossRef] [PubMed]

49. Tripathi, S.K.; Pandey, K.; Rengasamy, K.R.R.; Biswal, B.K. Recent updates on the resistance mechanisms to epidermal growth factor receptor tyrosine kinase inhibitors and resistance reversion strategies in lung cancer. Med. Res. Rev. 2020. [CrossRef] [PubMed]

50. Hong, S.P.; Chan, T.E.; Lombardo, Y.; Corleone, G.; Rotmensz, N.; Bravaccini, S.; Rocca, A.; Pruneri, G.; McEwen, K.R.; Coombes, R.C.; et al. Single-cell transcriptomics reveals multi-step adaptations to endocrine therapy. Nat. Commun. 2019, 10, 3840. [CrossRef] [PubMed]

51. Kagohara, L.T.; Zamuner, F.; Davis-Marcisak, E.F.; Sharma, G.; Considine, M.; Allen, J.; Yegnasubramanian, S.; Gaykalova, D.A.; Fertig, E.J. Integrated single-cell and bulk gene expression and ATAC-seq reveals heterogeneity and early changes in pathways associated with resistance to cetuximab in HNSCC-sensitive cell lines. Br. J. Cancer 2020, 123, 101-113. [CrossRef] [PubMed] 